PHYSICAL CHEMISTRY CHEMICAL PHYSICS (ISSN: 1463-9076) (eISSN: 1463-9084) 17: (38) pp. 24560-24565. (2015)

DOI: $10.1039 / \mathrm{C} 5 \mathrm{CP} 03153 \mathrm{~B}$ 


\title{
Glycosaminoglycans are potential pharmacological targets for classic DNA minor groove binder drugs berenil and pentamidine
}

\author{
Ferenc Zsila* \\ Research Group of Chemical Biology, Institute of Organic Chemistry, \\ Research Centre for Natural Sciences, Hungarian Academy of Sciences, POB 289, \\ H-1519, Budapest, Hungary
}

Corresponding author: Ferenc Zsila

POB 289, H-1519, Budapest, Hungary

Email: zsila.ferenc@tttk.mta.hu 


\begin{abstract}
It is shown that the antiprotozoal berenil and pentamidine, conventional minor groove binders of DNA, form non-covalent complexes with polyanionic glycosaminoglycans. Induced circular dichroism (CD) spectra as well as UV hypochromism confirmed drug binding to the asymmetric template of heparin and chondroitin 6-sulfate. The biphasic nature of the CD signals refer to intermolecular chiral exciton coupling between the dicationic guest molecules forming a right- or a left-handed helical array along the GAG chains. Quantitative evaluation of the spectroscopic data measured in $\mathrm{pH} 7.0$ buffer solution $(80 \mathrm{mM} \mathrm{NaCl})$ indicated a high- $\left(K_{\mathrm{a}} \sim 10^{6} \mathrm{M}^{-1}\right.$ for berenil) and a lower $\left(K_{\mathrm{a}} \sim 10^{5} \mathrm{M}^{-1}\right.$ for pentamidine) affinity heparin binding of these agents, similar to that reported for DNA. Drug-chondroitin sulfate complexes $\left(K_{\mathrm{a}} \sim 10^{4}-10^{5} \mathrm{M}^{-1}\right)$ could only be detected at low ionic strength. These results imply that besides nucleic acids, GAGs may be another pharmacological target for diarylamidine drugs.
\end{abstract}

Keywords: berenil; chondroitin sulfate, circular dichroism; exciton coupling; glycosaminoglycan; heparin, hypochromism; pentamidine

Abbreviations: CD, circular dichroism; CS, chondroitin 6-sulfate; GAG, glycosaminoglycan; ICD, induced circular dichroism; PNT, pentamidine 


\section{Introduction}

Pentamidine (PNT) is a symmetric diamidine derivative that was introduced in the 1940s to treat African trypanosomiasis and leishmaniasis. ${ }^{1,2}$ Later it was also shown to be effective against Pneumocystis carinii and today it is used commonly for treatment of AIDS-associated $P$. carinii infections. The polar benzamidine groups of PNT are connected through a conformationally mobile, central alkoxy chain (Scheme 1). Similarly to PNT, berenil (diminazene) also contains two benzamidine moieties but these are bridged by a shorter, sterically restricted triazene linker. As a highly active agent against a range of Trypanosoma species, berenil is approved for trypanosomiasis in domestic livestock. Although the anti-trypanosomal activity of berenil has also been documented in humans, it is presently registered for veterinary use only. ${ }^{3}$

PNT and berenil have been in the market for several decades but their mechanisms of action remains poorly understood. Since both compounds bind to the AT-rich sites of the minor groove of DNA, ${ }^{4,5}$ it was suggested that they might interfere with the nucleic acid biosynthesis. However, the DNA binding property does not account for the full spectrum of their pharmacological effects. ${ }^{2,6}$ In vitro data as well as animal models have showed that berenil possesses a variety of other biological actions such as enzyme activation and modulation of the host immune response. ${ }^{7}$ Taking into consideration the dicationic nature of these drugs, it can be hypothesized that the polyanionic, heterogeneously sulfated glycosaminoglycans (GAG) might be another macromolecular targets for them. These linear polysaccharides including heparin, heparan/chondroitin/dermatan/keratan sulfate are ubiquitous on many cell surfaces, in the extracellular matrix and also found 
intracellularly (e.g. in lysosomes). GAG chains are usually covalently attached to a core protein forming proteoglycans. ${ }^{8}$ Heparin is the highly sulfated species of heparan sulfate that occurs primarily as granules in mast cells but it is also present in normal human plasma at low concentration. ${ }^{9}$ GAGs interact non-covalently with a diverse set of protein ligands including chemo- and cytokines,${ }^{10}$ growth factors, ${ }^{11}$ enzymes, amyloid peptides, ${ }^{12}$ blood coagulation factors, and structural proteins. ${ }^{11}$ Through modulating the activities of protein partners, GAGs are involved in a number of normal and pathological processes such as cell-cell, virus-cell, cell-matrix adhesion, cell growth and differentiation, cell signaling, motility and anticoagulation. Besides the traditional protein and nucleic acid targets, pharmacological activities of small molecule drugs may also be mediated by perturbing GAG-protein binding. ${ }^{13}$ As far as the author is aware, no data have been reported either on PNT-GAG or berenil-GAG interactions. Therefore, employing circular dichroism (CD) and UV absorption spectroscopic methods, this work is aimed to detect and characterize the binding of PNT and berenil to GAG model compounds heparin and chondroitin 6-sulfate (CS). 


\section{Materials and methods}

\section{Materials}

Berenil (diminazene aceturate, cat\# sc-205651, purity 97.5\%) and pentamidine isethionate (cat\# sc-204176, purity $\geq 99 \%$ ) were obtained from Santa Cruz Biotechnology, Inc. Heparin sodium salt from porcine intestinal mucosa (Sigma, cat\# 51551), chondroitin 6-sulfate sodium salt from shark cartilage (Sigma, cat\# C4384) were used as supplied. All other reagents were of analytical grade.

\section{Preparation of drug and GAG solutions}

Stock solutions of drug and GAG samples were prepared freshly before each measurement in ultra-pure deionized water $(18.2 \mathrm{~m} \Omega)$ or in $50 \mathrm{mM}, \mathrm{pH} 7.0$ phosphate buffer containing $80 \mathrm{mM}$ sodium chloride. Due to the polydisperse nature of GAGs, their concentrations were calculated using the molecular weight of the average repeating disaccharide units: 665 and $503 \mathrm{~g} / \mathrm{mol}$ for heparin and CS, respectively.

\section{Circular dichroism and UV absorption spectroscopic measurements}

Spectroscopic experiments were conducted in ultra-pure deionized water $(18.2 \mathrm{~m} \Omega)$ or in $50 \mathrm{mM}, \mathrm{pH} 7.0 / 7.4$ phosphate buffer $(80 / 140 \mathrm{mM} \mathrm{NaCl})$ using a rectangular quartz cell of $1 \mathrm{~cm}$ optical path length (Hellma, USA). CD and absorption spectra were recorded on a JASCO J-715 spectropolarimeter at $25 \pm 0.2^{\circ} \mathrm{C}$ and represent the average of three scans obtained by collecting data at a scan speed of $100 \mathrm{~nm} / \mathrm{min}$. Temperature control was provided by a Peltier thermostat equipped with magnetic stirring. Absorption spectra 
were obtained by conversion of the high voltage $(\mathrm{HT})$ values of the photomultiplier tube of the CD equipment into absorbance units. CD and UV curves of drug-GAG mixtures were corrected by blank water or buffer solution. JASCO CD spectropolarimeters record CD data as ellipticity (' $\Theta$ ') in units of millidegrees (mdeg). The quantity of ' $\Theta$ ' is converted to molar circular dichroic absorption coefficient $\left(\Delta \varepsilon\right.$ in $\left.\mathrm{M}^{-1} \mathrm{~cm}^{-1}\right)$ using the equation $\Delta \varepsilon=\Theta /(33982 \mathrm{cl})$, where, 'c' is the molar concentration of the ligand $(\mathrm{mol} / \mathrm{L})$, and ' 1 ' is the optical pathlength expressed in $\mathrm{cm}$.

\section{Calculation of drug-GAG binding parameters}

Drug-GAG binding interactions can be quantified by the association constant $\left(K_{a}\right)$ :

$$
\mathrm{D}+\mathrm{GAG} \rightleftharpoons \mathrm{DGAG} ; \quad K_{a}=\frac{[D G A G]}{[D][G A G]}
$$

It is evident that

$$
\begin{gathered}
{[\mathrm{D}]=c_{\mathrm{d}}-[\mathrm{DGAG}]} \\
\text { and } \\
{[\mathrm{GAG}]=c_{\mathrm{gag}}-[\mathrm{DGAG}]}
\end{gathered}
$$

where $c_{d}$ and $c_{g a g}$ mean the total molar concentrations of the drug ligand and the GAG disaccharide units, respectively.

Since the formation of drug-GAG complexes is responsible for the UV hypochromism, it can be written that

$$
\varepsilon_{0}-\varepsilon=k[\mathrm{DGAG}] \text { or } \Theta_{\lambda}=k[\mathrm{DGAG}]
$$


where $\varepsilon_{0}$ and $\varepsilon$ are the molar absorption coefficients $\left(\mathrm{M}^{-1} \mathrm{~cm}^{-1}\right)$ of the main UV peak of the drug measured in the absence and presence of the GAG, $\Theta_{\lambda}$ is the induced ellipticity value (mdeg) at a given wavelength, and $k$ is a constant.

Using equations $1-4$, we obtain

$$
\begin{gathered}
\left(\varepsilon_{0}-\varepsilon\right)=\frac{k}{2}\left(c_{d}+c_{g a g}+K_{a}^{-1}-\sqrt{\left(c_{d}+c_{g a g}+K_{a}^{-1}\right)^{2}-4 c_{d} c_{g a g}}\right) \\
\text { and } \\
\Theta_{\lambda}=\frac{k}{2}\left(c_{d}+c_{g a g}+K_{a}^{-1}-\sqrt{\left(c_{d}+c_{g a g}+K_{a}^{-1}\right)^{2}-4 c_{d} c_{g a g}}\right)
\end{gathered}
$$

Non-linear regression analysis was applied to calculate the apparent value of $K_{a}$ using the NLREG ${ }^{\circledR}$ software (statistical analysis program, version 3.4). The number of drug molecules bound per a disaccharide unit $(n)$ was estimated by adjusting the drug concentration $\left(c_{d}\right)$ during the curve fitting procedure. For instance, the best curve fit on the UV data $\left(\varepsilon_{0}-\varepsilon\right)$ obtained by titration of berenil with heparin was achieved by using $14 \mu \mathrm{M}$ instead of the experimental value of $49 \mu \mathrm{M}$. The ratio of $49 / 14$ gives the average number of berenil molecules bound per a disaccharide unit (Supplementary Fig. 1). 


\section{Results and discussion}

The UV band of berenil centered at $369 \mathrm{~nm}$ in $\mathrm{pH} 7.0$ buffer solution showed decreasing absorbance (hypochromism) and $5 \mathrm{~nm}$ blue shift upon addition of heparin (Fig. 1). The absorption curves show an isosbestic point at $413 \mathrm{~nm}$ suggesting an equilibrium between the free and heparin bound form of the drug molecules. Though it lacks intrinsic CD activity, berenil displayed strong induced CD (ICD) signals in the presence of heparin. The oppositely signed band pair consists of a more intense, longer-wavelength positive and a shorter-wavelength negative peak with a zero cross-over point around $368 \mathrm{~nm}$ that coincides with the $\lambda_{\max }$ of the respective absorption band (Fig. 1). Shape, position and intensity ratio of the ICD peaks were invariant to the increase of heparin/berenil molar ratio. These $\mathrm{CD}$ and absorption spectroscopic features are characteristic to the intermolecular exciton coupling between the long axis polarized $\pi-\pi^{*}$ transition moments of berenil molecules accommodated close to each other along the heparin chain. ${ }^{14,15}$ Chiral disposition of the drug molecules are dictated by the helically arranged sulphate/carboxylate groups to which they are attached via ionic and/or H-bonds. Employing the exciton coupling theory, ${ }^{16,17}$ the positive-negative band $\mathrm{CD}$ signature predicts the right-handed ( $P$-helical) intermolecular orientation of the long axes of adjacent drug molecules bound consecutively to the anionic sites of asymmetric heparin template (Fig. 2).

Non-linear regression analysis of the absorption and CD spectroscopic changes measured by titrating a berenil solution with heparin resulted in a high-affinity $\left(K_{\mathrm{a}} \sim 10^{6} \mathrm{M}^{-1}\right)$ binding (Supplementary Fig. 1, Table 1). Importantly, the DNA association constant of 
berenil is also $\sim 10^{6} \mathrm{M}^{-1}$ indicating comparable affinity of berenil for heparin and DNA. ${ }^{5,18}$ According to the stoichiometry of the binding (n), an average of three drug molecules are associated to a repeating disaccharide unit that is close to its negative charge density (Table 1). The CD and UV spectra measured in water with $100 \mathrm{mM} \mathrm{NaCl}$ (Supplementary Fig. 2) as well as the corresponding binding parameters were close to that obtained in buffer solution (Table 1). These results imply that mild acidic conditions do not affect significantly the berenil-heparin interaction.

Of notice, at physiological $\mathrm{pH}$ and $\mathrm{Na}^{+}$concentration $(140 \mathrm{mM})$ berenil molecules also associate to the heparin chain producing similar but less intense CD and absorption spectroscopic changes to that seen in deionized water and in $\mathrm{pH} 7$ buffer solution at lower ionic strength (Supplementary Fig. 3). Furthermore, the ICD signals are time-dependent at physiological sodium ion level: instead of a prompt increase, the ellipticity values slowly raise in time after addition of the heparin aliquots. This may be accounted for by the cationic perturbation of the electrostatic interactions between berenil and the anionic heparin sites.

CS induced a heparin-like bisignate, but only a half-intensity CD band pair for berenil (Fig. 3). In relation to the change of the $\lambda_{\max }$ value obtained with heparin (Fig. 1), a larger blue shift $(9 \mathrm{~nm})$ was observed. In the frame of the exciton theory, ${ }^{16,17}$ this difference is indicative for the decrease of the overlay angle between the long axes of adjacent berenil molecules associated to the stereochemically distinct binding sites of CS (Fig. 2). However, the ICD feature as well as the UV blue shift and hypochromism could only be observed in deionized water. Increase of the $\mathrm{Na}^{+}$concentration rapidly canceled the ICD signals and restored the absorption spectroscopic profile characteristic to the free drug 
molecules (Supplementary Fig. 4). These findings suggest that in marked contrast to heparin, CS binding of berenil can easily be disrupted by sodium ions and thus it may strongly suppressed in a physiological ionic milieu. It should also be noted that the CS binding affinity of berenil calculated from the CD and UV spectroscopic changes is 100 times lower than that of heparin (Table 1).

Binding of PNT to CS in aqueous solution gives rise to negative-positive ICD peaks and a small UV hypochromism (Fig. 4). The zero line intersection of the ICD curve at $263 \mathrm{~nm}$ is very close to the slightly red shifted absorption maximum. Compared to berenil, the less intense hypochromism suggests a greater steric spacing between the interacting benzamidine chromophores. Furthermore, the red shift of the absorption peak is consistent with a steric disposition where the angle between the interacting transition dipole moments is greater that $90^{\circ}$ (Fig. 2). ${ }^{16,17}$ As it follows from the negative-positive ellipticity feature, long axes of the exciton coupled benzamidine moieties are twisted in a counterclockwise manner (left-handed or $M$-helical orientation, Fig. 2). Similarly to the berenil-CS interaction, both the CD as well as the absorption spectroscopic changes were completely reversible on a small increase of the sodium concentration (Supplementary Fig. 5).

Due to the flexibility of the saturated dioxypentane linker, PNT molecules may adopt a chiral conformation when bind to CS within which the benzamidine moieties come close to each other. Therefore, the induced exciton CD couplet (Fig. 4) may also be of intramolecular origin. ${ }^{17,19}$ To discriminate between the intra- and intermolecular coupling, PNT was added to the high molar excess of CS. Under such condition, the drug molecules are well separated from each other along the GAG chains and thus not inter- 
but only intramolecular exciton coupling can occur. The CD inactivity measured for this sample (Supplementary Fig. 6) lends credence to the intermolecular exciton mechanism that is further supported by multimeric $(n \sim 3)$ binding of the drug molecules (Table 1$)$.

In relation to $\mathrm{CS}$, the spectroscopic data indicate that PNT can bind to heparin even at much higher ionic strength $(\mathrm{pH} 7.0$ phosphate buffer, $80 \mathrm{mM} \mathrm{NaCl}$ ) displaying a somewhat larger hypochromism and a positive-negative ICD couplet (Fig. 5). The mirror image relation of the exciton coupling pattern of PNT-CS (Fig. 4) and PNT-heparin (Fig. 5) complexes suggests the opposite intermolecular chiral organization of the drug molecules (left- and right-handed) associated to the GAG chains (Fig. 2).

Raising the sodium concentration in the sample to physiological level cancels the ICD signals of PNT but a small UV hypochromism still can be observed in the presence of heparin (Supplementary Fig. 7). The physiological sodium concentration applied here $(140 \mathrm{mM})$ is characteristic to the extracellular space. However, intracellular organelles such as lysosomes are featured with lower cationic content, $\sim 60 \mathrm{mM} \mathrm{Na}^{+}$and $\sim 20 \mathrm{mM}$ $\mathrm{K}^{+},{ }^{20}$ that is very close to the ionic strength used in the present study $\left(80-100 \mathrm{mM} \mathrm{Na}{ }^{+}\right.$, see Table 1). Importantly, catabolism of GAGs is intimately related to lysosomes ${ }^{21}$ and pentamidine was shown to be selectively accumulated in these subcellular compartments. $^{22}$ Accordingly, by non-covalent association to GAGs, pentamidine (and presumably berenil) may perturb lysosome function. ${ }^{23}$

In comparison to berenil, evaluation of the UV titration data showed ten times weaker heparin affinity of PNT (Table 1) that may be accounted for by structural differences. Besides the terminal amidinium groups, the central triazene moiety of berenil can form an additional intermolecular $\mathrm{H}$-bond resulting in tighter interaction. Nevertheless, the 
heparin association constant of PNT $\left(\sim 10^{5} \mathrm{M}^{-1}\right)$ is close to that proposed for its DNA binding. ${ }^{6}$

Very recently, the heparin association of the bis-4-aminoquinoline surfen has been evaluted using CD and UV spectroscopic methods. ${ }^{19}$ Though the biphasic ICD spectrum of surfen-heparin complexes is reminiscent to that of berenil, it stems from intra- and not intermolecular exciton coupling mechanism. Distinctly from the planar, sterically restricted berenil molecule, surfen can bind to the heparin sites in a helically twisted (right-handed) conformation due to the rotation of the aminoquinoline moieties around the central urea core. Similarly to berenil, however, higher drug loading of the heparin chains results in surfen-surfen intermolecular exciton coupling associated with strong UV hypochromism. ${ }^{19}$ In comparison to berenil, the heparin affinity of surfen is ten-times higher $\left(\sim 10^{7} \mathrm{M}^{-1}\right)$ that may be related to its ability to adopt a crescent-shaped conformation, where the two amino groups can form multiple H-bonds with a sulfate substituent of the heparin template. ${ }^{14}$ 


\section{Conclusions}

The spectroscopic results described herein show that conventional DNA binding diamidine drugs can also bind to polyanionic GAG molecules with similar affinity. Interaction of berenil and pentamidine with the helically arranged sulfate and carboxylate groups of heparin and chondroitin 6-sulfate chains allows their multimeric, chiral association to the disaccharide units. In contrast to CS, the higher charge density of heparin favours the tighter association of these cationic agents. The GAG binding ability demonstrated here suggests that berenil and pentamidine may interfere with functional GAG-protein interactions providing a so far overlooked molecular pathway to better understand their pharmacological actions.

Electronic supplementary information (ESI) available: additional CD and absorption spectra. 


\section{References}

1. M. d. N. C. Soeiro, E. M. d. Souza and D. W. Boykin, Expert Opin. Ther. Pat., 2007, 17, 927-939.

2. M. Sands, M. A. Kron and R. B. Brown, Rev. Infect. Dis., 1985, 7, 625-634.

3. A. S. Peregrine and M. Mamman, Acta Trop., 1993, 54, 185-203.

4. D. S. Pilch, M. A. Kirolos, X. Liu, G. E. Plum and K. J. Breslauer, Biochemistry, 1995, 34, 9962-9976.

5. T. C. Jenkins, A. N. Lane, S. Neidle and D. G. Brown, Eur. J. Biochem., 1993, 213, $1175-1184$.

6. T. Sun and Y. Zhang, Nucleic Acids Res., 2008, 36, 1654-1664.

7. S. Kuriakose and J. E. Uzonna, Int. Immunopharmacol., 2014, 21, 342-345.

8. J. Halper, Adv. Exp. Med. Biol., 2014, 802, 49-58.

9. N. Volpi, M. Cusmano and T. Venturelli, Biochim. Biophys. Acta, 1995, 1243, 49-58.

10. I. Kufareva, C. L. Salanga and T. M. Handel, Immunol. Cell Biol., 2015, 93, 372-383.

11. D. Xu and J. D. Esko, Annu. Rev. Biochem., 2014, 83, 129-157.

12. H. Cui, C. Freeman, G. A. Jacobson and D. H. Small, IUBMB Life, 2013, 65, 108-120.

13. N. Harris, F. Y. Kogan, G. Il'kova, S. Juhas, O. Lahmy, Y. I. Gregor, J. Koppel, R. Zhuk and P. Gregor, Biochim. Biophys. Acta, 2014, 1840, 245-254.

14. F. Zsila, Biochem. Biophys. Res. Commun., 2015, 460, 863-867.

15. F. Zsila and G. Gedeon, Biochem. Biophys. Res. Commun., 2006, 346, 1267-1274.

16. S. E. Boiadjiev and D. A. Lightner, Monatsh. Chem., 2005, 136, 489-508.

17. N. Berova, D. Gargiulo, F. Derguini, K. Nakanishi and N. Harada, J. Am. Chem. Soc., 1993, 115, 4769-4775.

18. C. A. Laughton, F. Tanious, C. M. Nunn, D. W. Boykin, W. D. Wilson and S. Neidle, Biochemistry, 1996, 35, 5655-5661.

19. F. Zsila, Chirality, 2015, in press, DOI: 10.1002/chir.22471.

20. B. E. Steinberg, K. K. Huynh, A. Brodovitch, S. Jabs, T. Stauber, T. J. Jentsch and S. Grinstein, J. Cell Biol., 2010, 189, 1171-1186. 
21. M. F. Coutinho, L. Matos and S. Alves, Gene, 2015, 555, 50-58.

22. H. Glaumann, U. Bronner, O. Ericsson, L. L. Gustafsson and L. Rombo, Pharmacol. Toxicol., 1994, 74, 17-22.

23. J. Fischer, H. Lullmann and R. Lullmann-Rauch, Gen. Pharmacol., 1996, 27, $1317-$ 1324. 


\begin{tabular}{|c|c|c|c|c|c|}
\hline Drug & $\boldsymbol{K}_{\mathbf{a}}\left(\mathrm{M}^{-1}\right)$ & $n$ & Solvent & $\begin{array}{c}{\left[\mathrm{Na}^{+}\right]} \\
\mathrm{mM}\end{array}$ & Method \\
\hline \multirow{4}{*}{ Berenil-HP } & $1.1( \pm 0.3) \times 10^{6}$ & 3.5 & $\mathrm{pH} 7.0$ & \multirow{2}{*}{80} & \\
\hline & $1.1( \pm 0.1) \times 10^{6}$ & 2.7 & buffer & & $\mathrm{CD}(388 \mathrm{~nm})$ \\
\hline & $1.1( \pm 0.1) \times 10^{6}$ & 3.4 & \multirow{2}{*}{$\mathrm{H}_{2} \mathrm{O}$} & \multirow{2}{*}{100} & UV \\
\hline & $1.0( \pm 0.2) \times 10^{6}$ & 2.6 & & & $\mathrm{CD}(388 \mathrm{~nm})$ \\
\hline \multirow{2}{*}{ Berenil-CS } & $2.7( \pm 0.3) \times 10^{4}$ & 2.4 & \multirow{2}{*}{$\mathrm{H}_{2} \mathrm{O}$} & \multirow{2}{*}{0} & UV \\
\hline & $1.7( \pm 0.4) \times 10^{4}$ & 2.4 & & & $\mathrm{CD}(383 \mathrm{~nm})$ \\
\hline \multirow{2}{*}{ PNT-HP } & $1.6( \pm 0.1) \times 10^{5}$ & 3.2 & $\begin{array}{l}\text { pH } 7.0 \\
\text { buffer }\end{array}$ & 80 & \multirow{2}{*}{ UV } \\
\hline & $2.3( \pm 0.1) \times 10^{5}$ & 4.3 & $\mathrm{H}_{2} \mathrm{O}$ & 80 & \\
\hline PNT-CS & $1.0( \pm 0.1) \times 10^{5}$ & 3.2 & $\mathrm{H}_{2} \mathrm{O}$ & 0 & UV \\
\hline
\end{tabular}

\section{Table 1}

Heparin (HP) and chondroitin 6-sulfate (CS) association constants and number of the binding sites per a disaccharide unit $(n)$ of berenil and pentamidine (PNT) estimated from UV and CD spectroscopic titration data. 

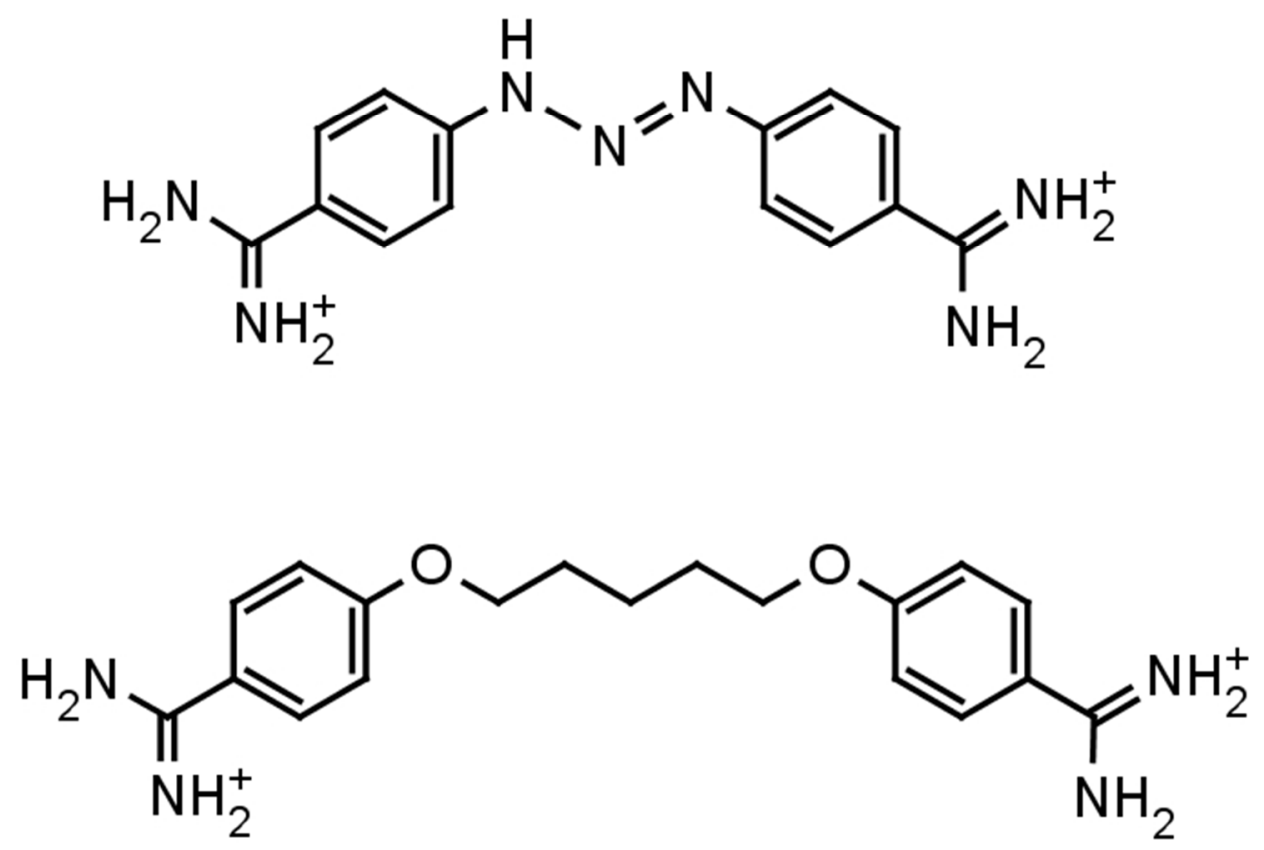

Scheme 1

Chemical structures of the cationic form of berenil (top) and pentamidine. 

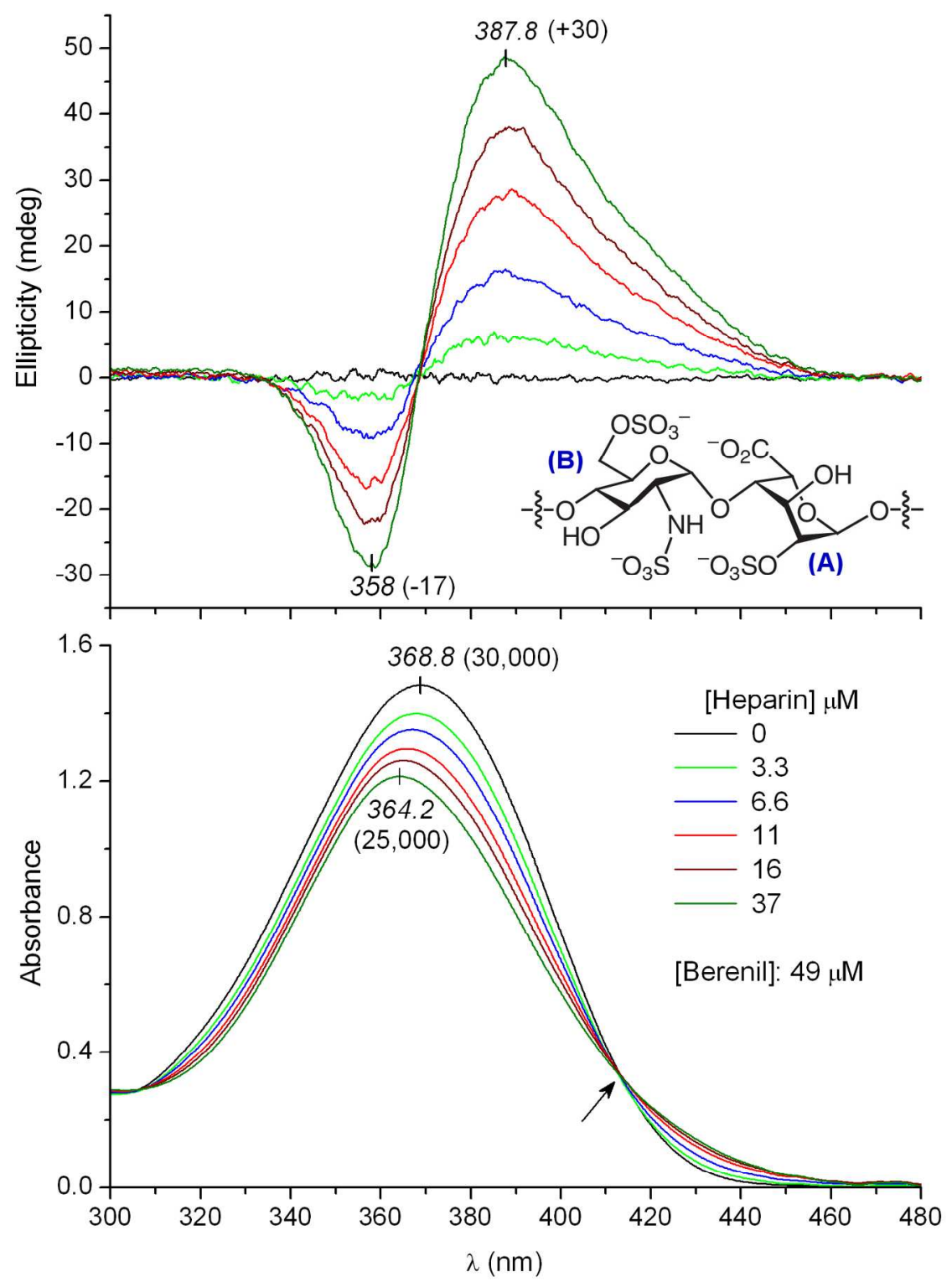

Figure 1

Induced $\mathrm{CD}$ and absorption spectra of berenil at increasing concentrations of heparin ( 50 mM phosphate buffer at $\mathrm{pH} 7.0,80 \mathrm{mM} \mathrm{Na}^{+1}, 25^{\circ} \mathrm{C}$ ). Molar absorption $(\varepsilon)$ and circular dichroic absorption cofficients $( \pm \Delta \varepsilon)$ calculated by using the concentration of berenil are shown in parentheses. Arrow denotes an isosbestic point at $413 \mathrm{~nm}$. Inset displays the repeating disaccharide unit of heparin composed of L-iduronic acid 2-O-sulfate (A) and $\mathrm{D}$ - $\mathrm{N}$-sulfoglucosamine 6-O-sulfate (B). $635 \times 847 \mathrm{~mm}(72 \times 72$ DPI $)$ 

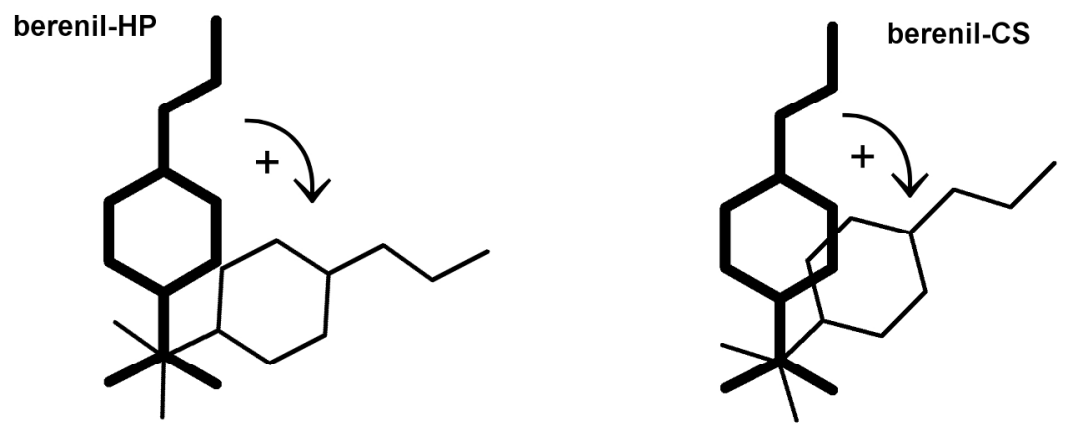

UV spectrum: blue shift (with HP) < blue shift (with CS) CD spectrum: positive-negative exciton couplet Intermolecular chirality: right-handed or P-helical

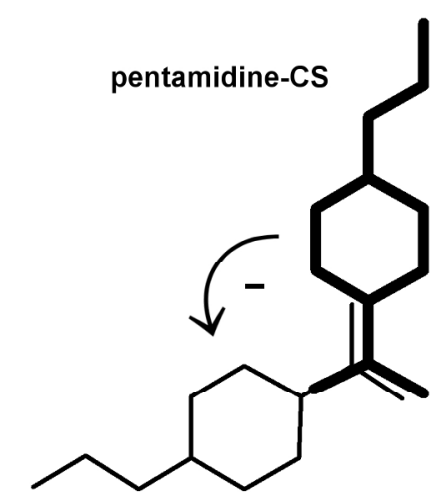

UV spectrum: red shift

CD spectrum: negative-positive exciton couplet Intermolecular chirality: left-handed or $M$-helical

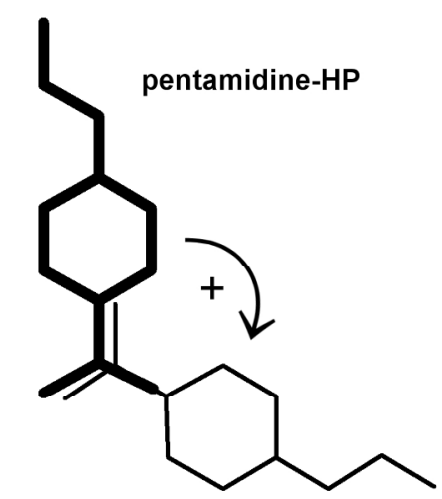

UV spectrum: red shift

CD spectrum: positive-negative exciton couplet Intermolecular chirality: right-handed or $P$-helical

Figure 2

Schematic representation of the intermolecular chiral arrangements of the benzamidine moieties of two adjacent drug molecules bound to heparin (HP) and chondroitin 6-sulfate (CS). Thickened bonds are lying above the plane of the paper. Arrows denote the sense of the chiral twist (clockwise-positive, counterclockwise-negative) between the coupled $\Pi-\Pi^{*}$ transition moments oriented along the long axis of the benzamidine chromophores.

$840 \times 839 \mathrm{~mm}(72 \times 72$ DPI $)$ 

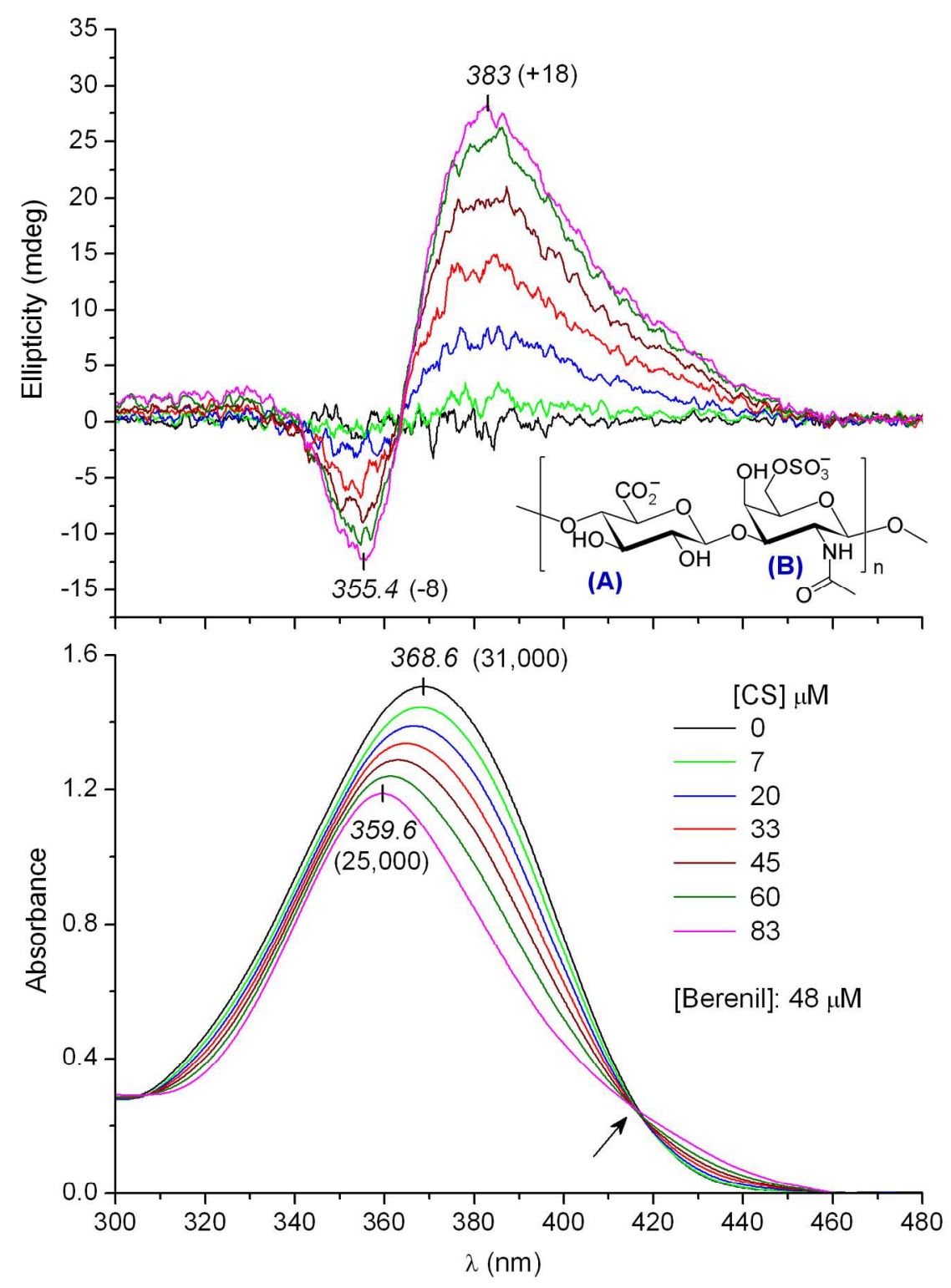

Figure 3

Induced $\mathrm{CD}$ and absorption spectra of berenil at increasing concentrations of $\mathrm{CS}$ in deionized water ( $\left.25^{\circ} \mathrm{C}\right)$. Molar absorption $(\varepsilon)$ and circular dichroic absorption cofficients $( \pm \Delta \varepsilon$ ) calculated by using the concentration of berenil are shown in parentheses. Arrow denotes an isosbestic point at $416 \mathrm{~nm}$. Inset displays the repeating disaccharide unit of chondroitin 6-sulfate composed of $\beta$-glucuronic acid (A) and $N$-acetyl- $\beta$ galactosamine-6-sulfate $(B)$. $635 \times 850 \mathrm{~mm}(72 \times 72 \mathrm{DPI})$ 


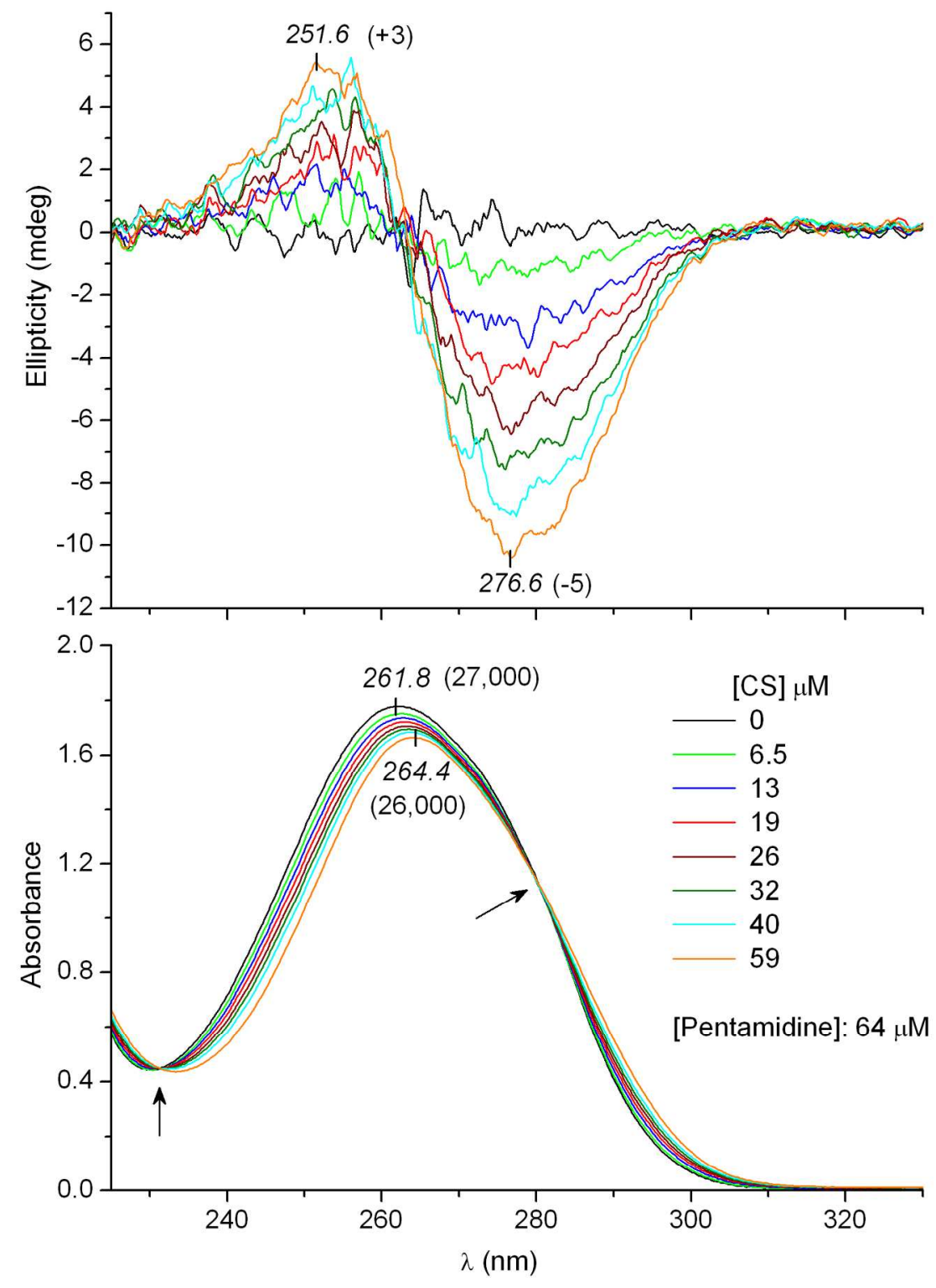

Figure 4

Induced $C D$ and absorption spectra of pentamidine measured at increasing concentrations of CS in deionized water $\left(25^{\circ} \mathrm{C}\right)$. Molar absorption $(\varepsilon)$ and circular dichroic absorption cofficients $( \pm \Delta \varepsilon)$ calculated by using drug concentration are shown in parentheses. Arrows denote isosbestic points at 231 and $280 \mathrm{~nm}$. $635 \times 862 \mathrm{~mm}(72 \times 72 \mathrm{DPI})$ 

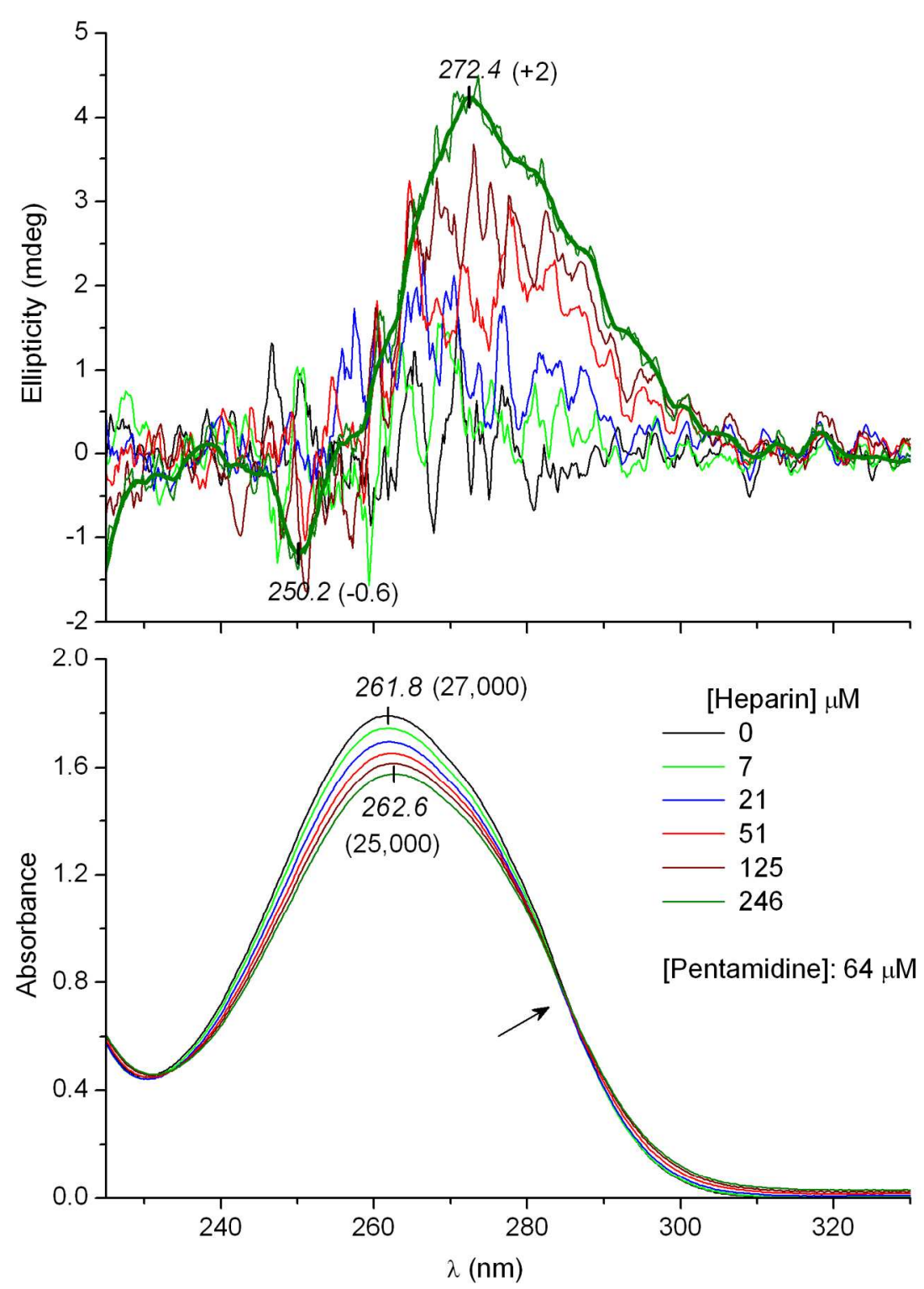

Figure 5

Induced $C D$ and absorption spectra of pentamidine measured at increasing concentrations of heparin (50 $\mathrm{mM}$ phosphate buffer at $\mathrm{pH} 7.0,80 \mathrm{mM} \mathrm{Na}+, 25^{\circ} \mathrm{C}$ ). Molar absorption $(\varepsilon)$ and circular dichroic absorption cofficients $( \pm \Delta \varepsilon)$ calculated by using drug concentration are shown in parentheses. Thin line shows the smoothed ICD curve of PNT measured at $246 \mu \mathrm{M}$ heparin. Arrow denotes an isosbestic point at $285 \mathrm{~nm}$. $631 \times 872 \mathrm{~mm}(72 \times 72 \mathrm{DPI})$ 


\section{Supplementary Information}




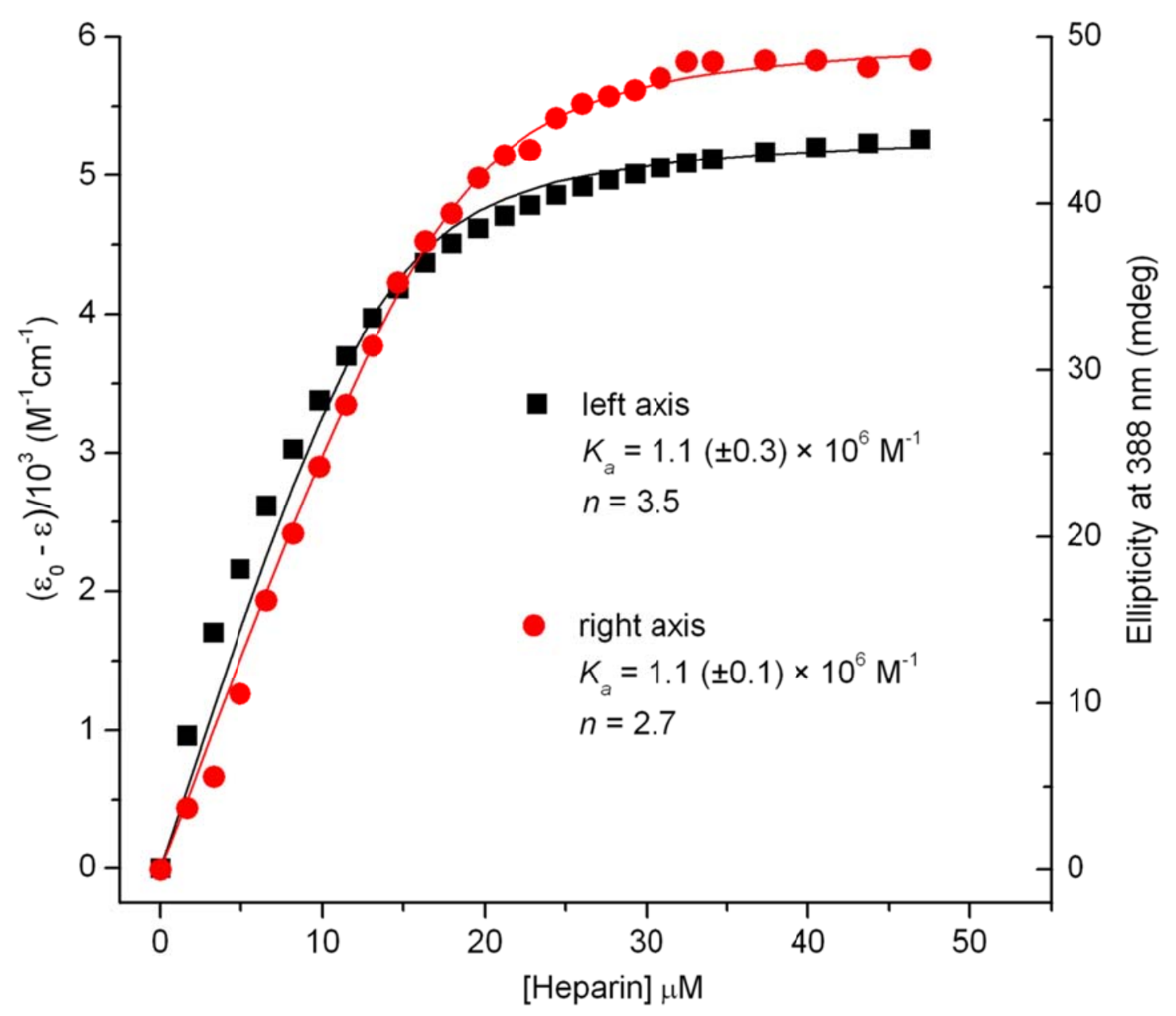

\section{Supplementary Figure 1}

UV absorption changes and induced CD values of berenil (49 $\mu \mathrm{M})$ plotted against the heparin concentration of the sample solution (50 $\mathrm{mM}$ phosphate buffer at $\mathrm{pH} 7.0,80 \mathrm{mM} \mathrm{Na}^{+}, 25^{\circ} \mathrm{C}$ ). $\varepsilon_{0}$ : maximum molar absorption coefficient of the main UV peak of berenil measured in heparin-free buffer solution; $\varepsilon$ : molar absorption coefficient measured at increasing concentrations of heparin. Solid lines are the results of non-linear curve fitting analysis. Estimated association constant $\left(K_{\mathrm{a}}\right)$ and the number of berenil binding sites $(n)$ per a disaccharide unit are shown. 

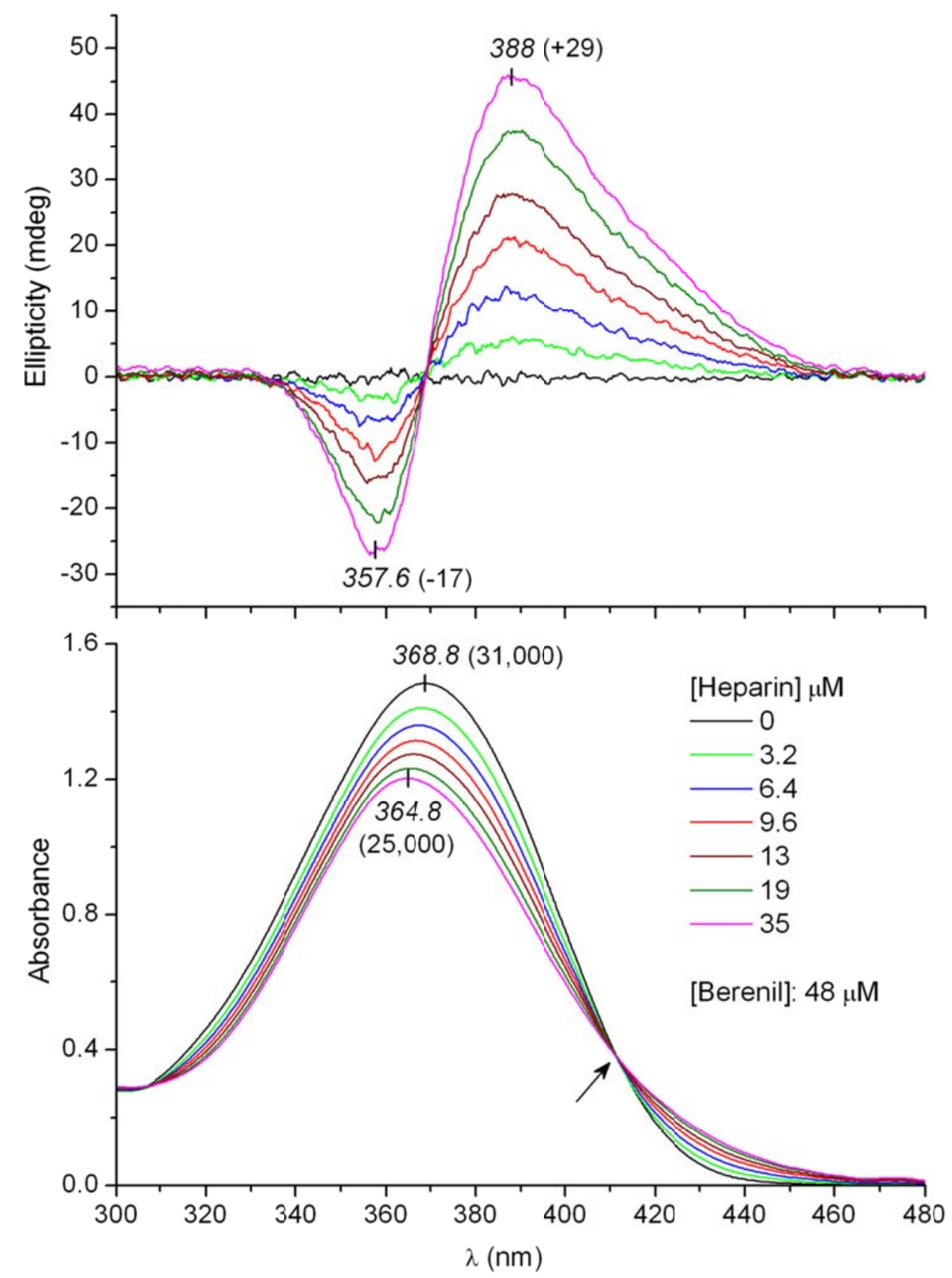

\section{Supplementary Figure 2}

Induced CD and absorption spectra of $48 \mu \mathrm{M}$ berenil at increasing concentrations of heparin in deionized water (100 mM NaCl, $\left.25^{\circ} \mathrm{C}\right)$. Molar absorption ( $(\varepsilon)$ and circular dichroic absorption cofficients $( \pm \Delta \varepsilon)$ calculated by using the concentration of berenil in the sample solution are shown in parentheses. Arrow denotes an isosbestic point at $411 \mathrm{~nm}$. 


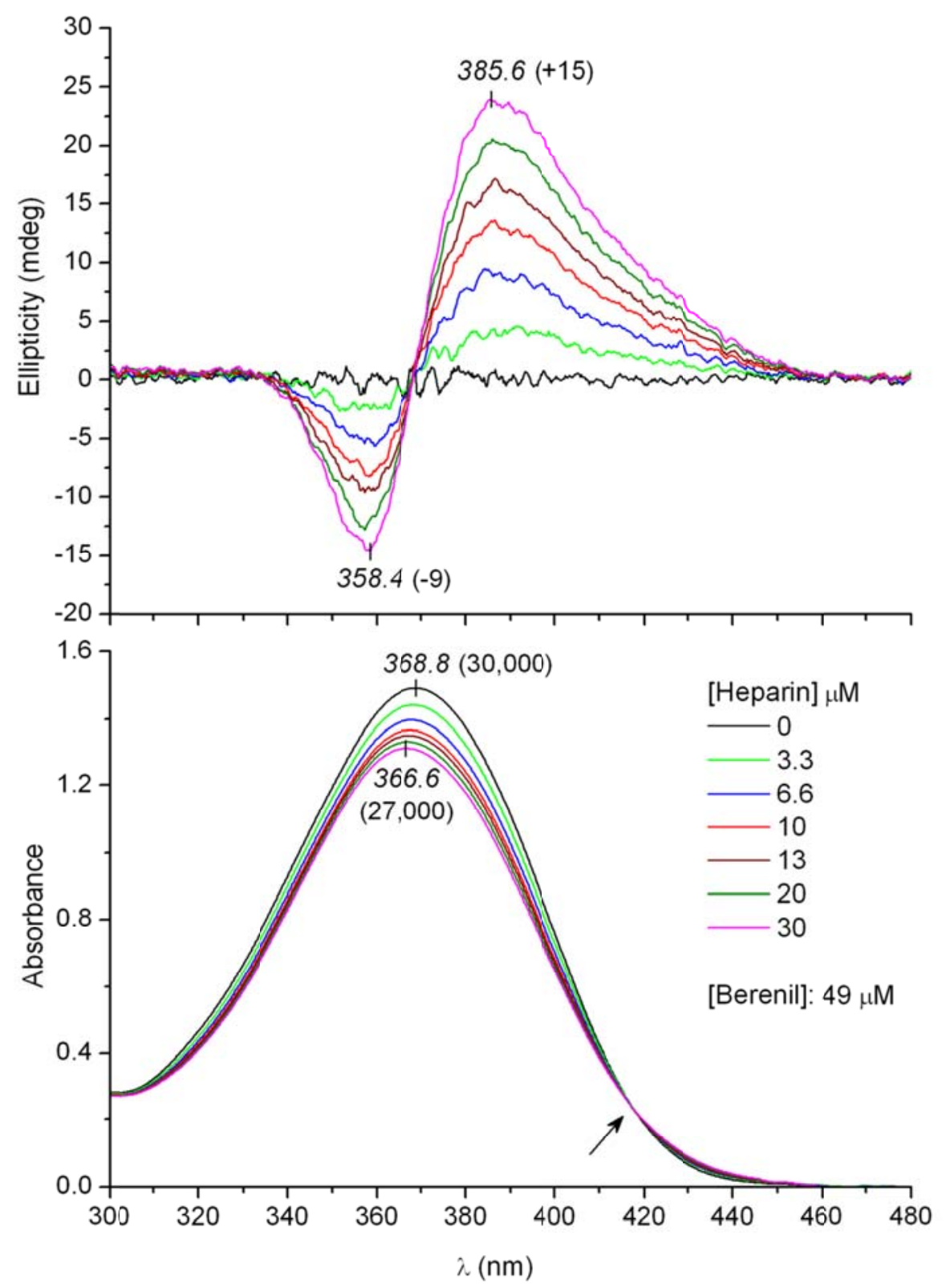

\section{Supplementary Figure 3}

Induced CD and absorption spectra of $49 \mu \mathrm{M}$ berenil at increasing concentrations of heparin in $\mathrm{pH} 7.4$ phosphate buffer with $140 \mathrm{mM} \mathrm{Na}^{+}\left(25^{\circ} \mathrm{C}\right)$. Molar absorption $(\varepsilon)$ and circular dichroic absorption cofficients $( \pm \Delta \varepsilon)$ calculated by using the concentration of berenil in the sample solution are shown in parentheses. Due to the time-dependence of the CD curves, each scan was performed with 15 min. delay after the addition of heparin aliquots. Arrow denotes an isosbestic point at $417 \mathrm{~nm}$. 

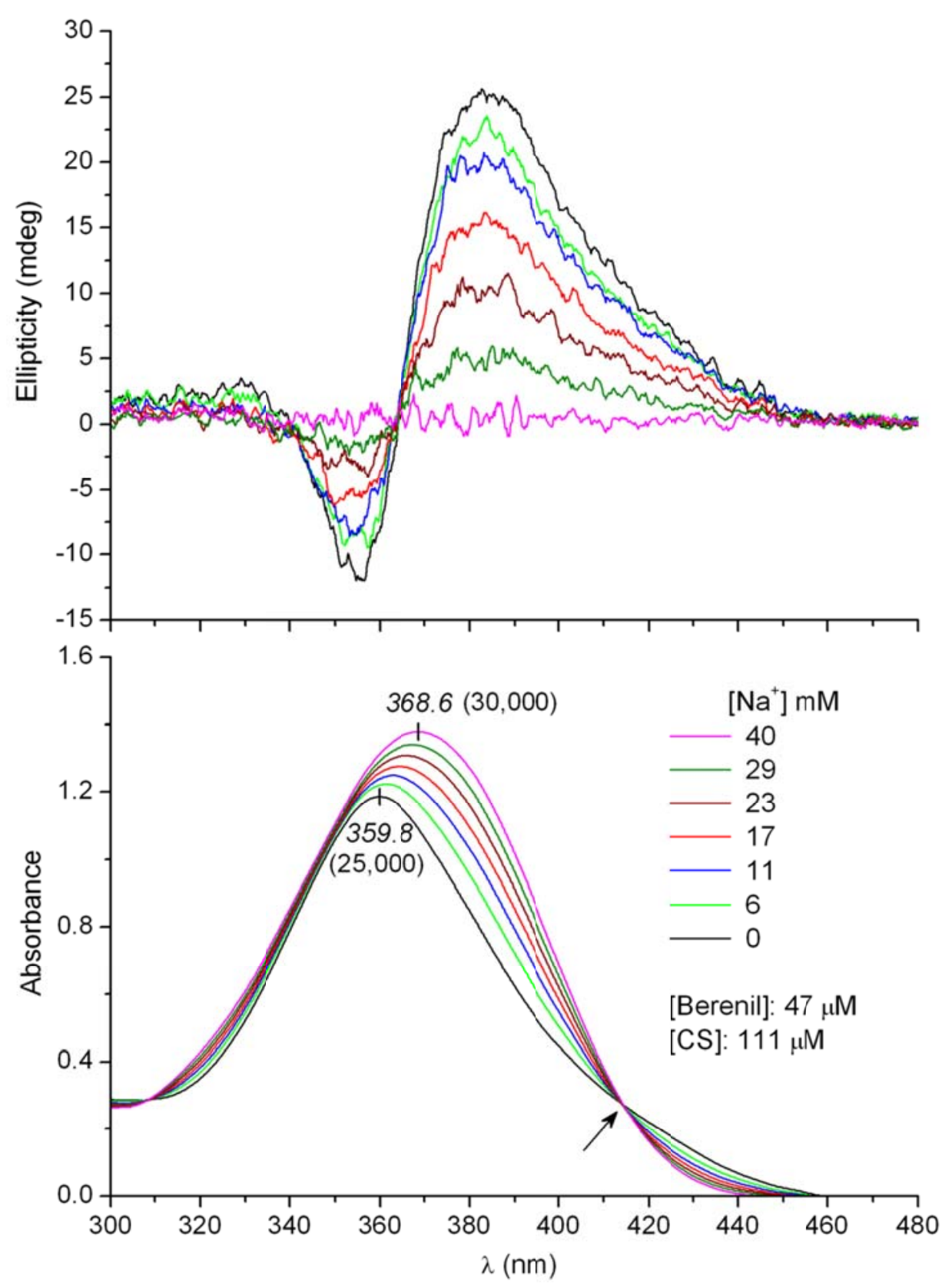

\section{Supplementary Figure 4}

Effect of increasing sodium ion concentration on the ICD and UV absorption spectrum of berenil in CS solution (deionized water, $25^{\circ} \mathrm{C}$ ). Molar absorption cofficients ( $\varepsilon$ ) of berenil are shown in parentheses. Arrow denotes an isosbestic point at $414 \mathrm{~nm}$. 

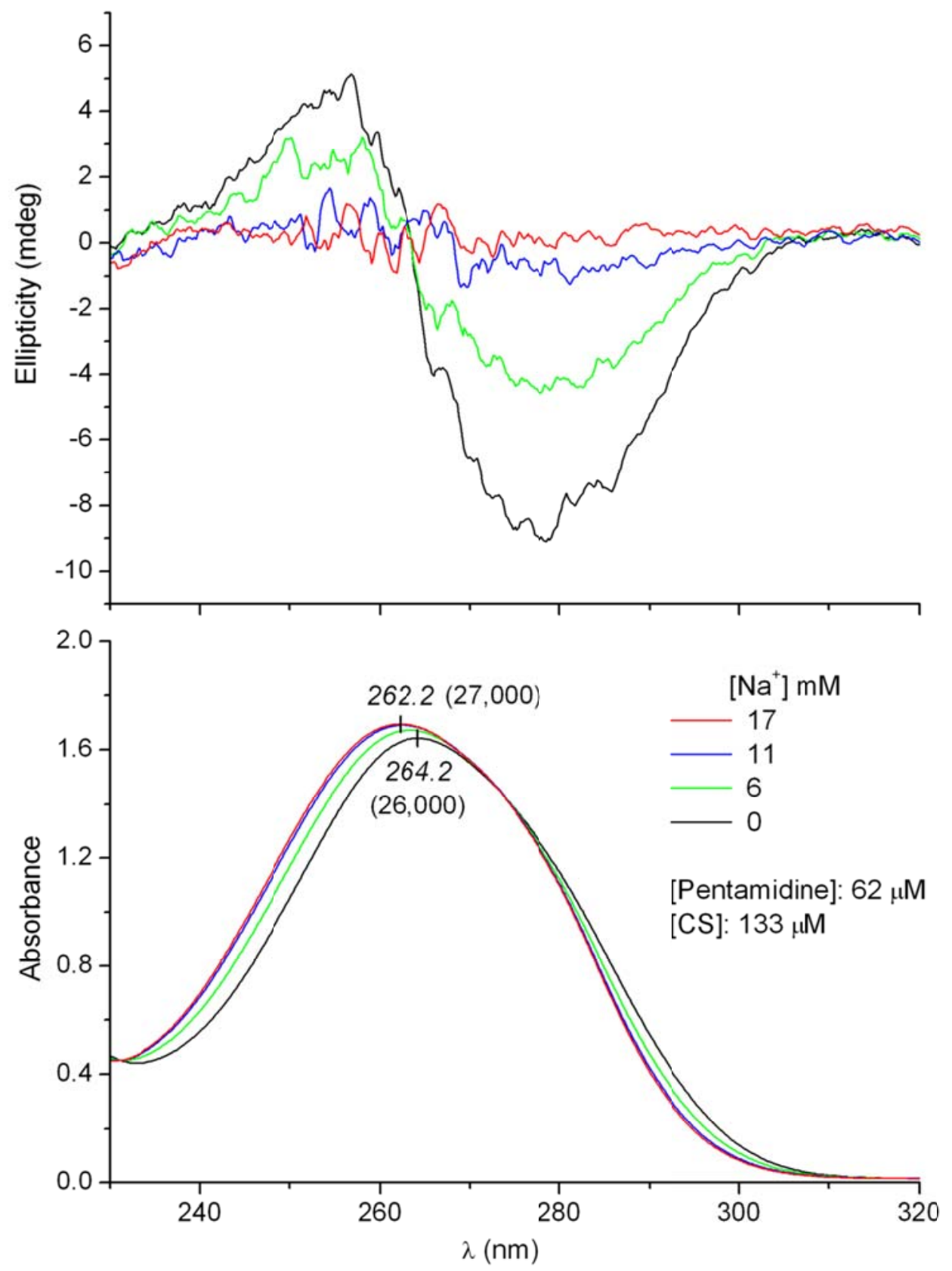

\section{Supplementary Figure 5}

Effect of increasing sodium ion concentration on the ICD and UV absorption spectrum of pentamidine in chondroitin 6-sulfate solution (deionized water, $25^{\circ} \mathrm{C}$ ). Molar absorption cofficients $(\varepsilon)$ of the drug are shown in parentheses. 


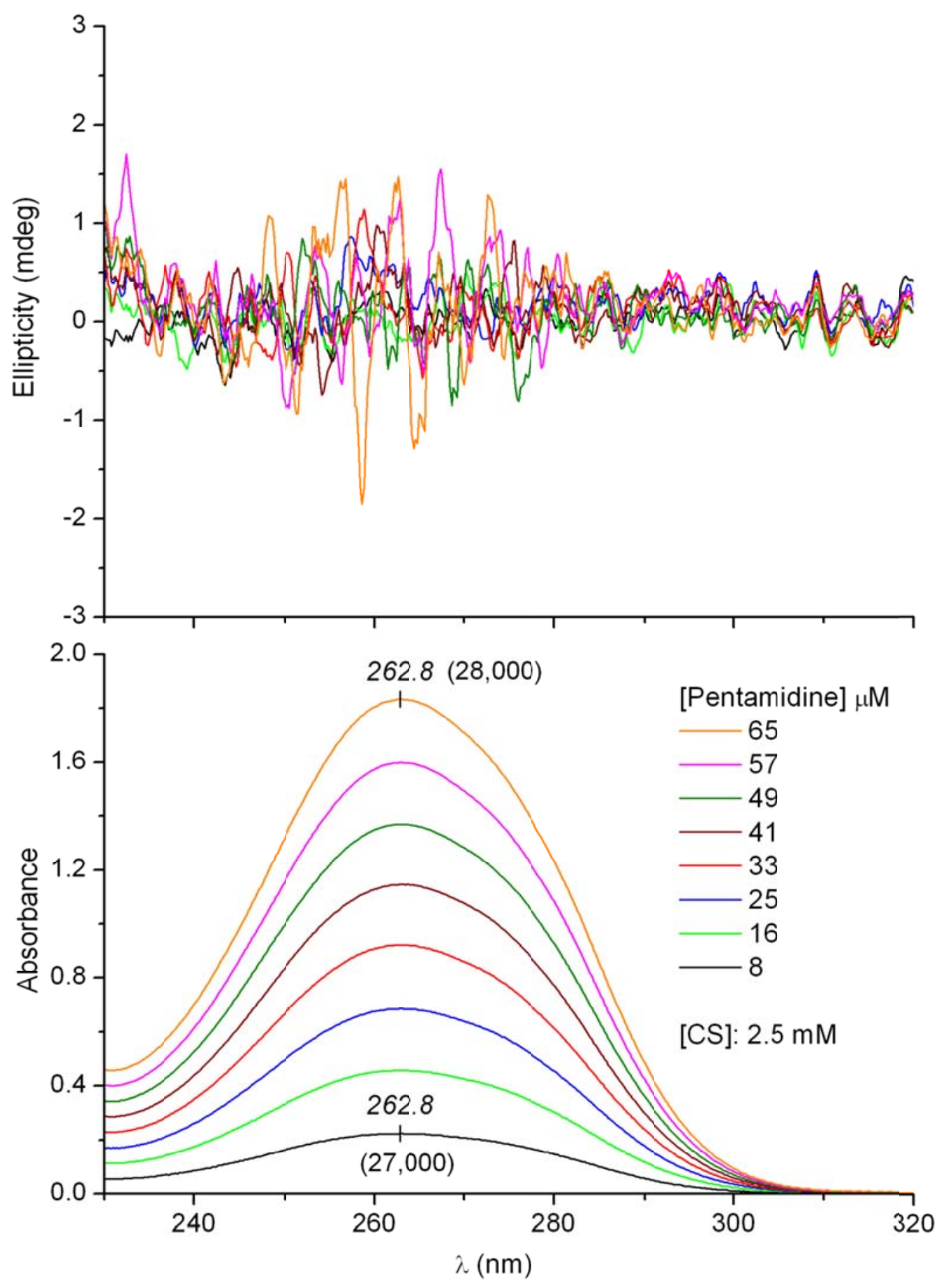

\section{Supplementary Figure 6}

$\mathrm{CD}$ and absorption spectra of pentamidine in aqueous solution of chondroitin 6-sulfate (25 $\left.{ }^{\circ} \mathrm{C}\right)$. Molar absorption cofficients ( $\varepsilon$ ) are shown in parentheses. 

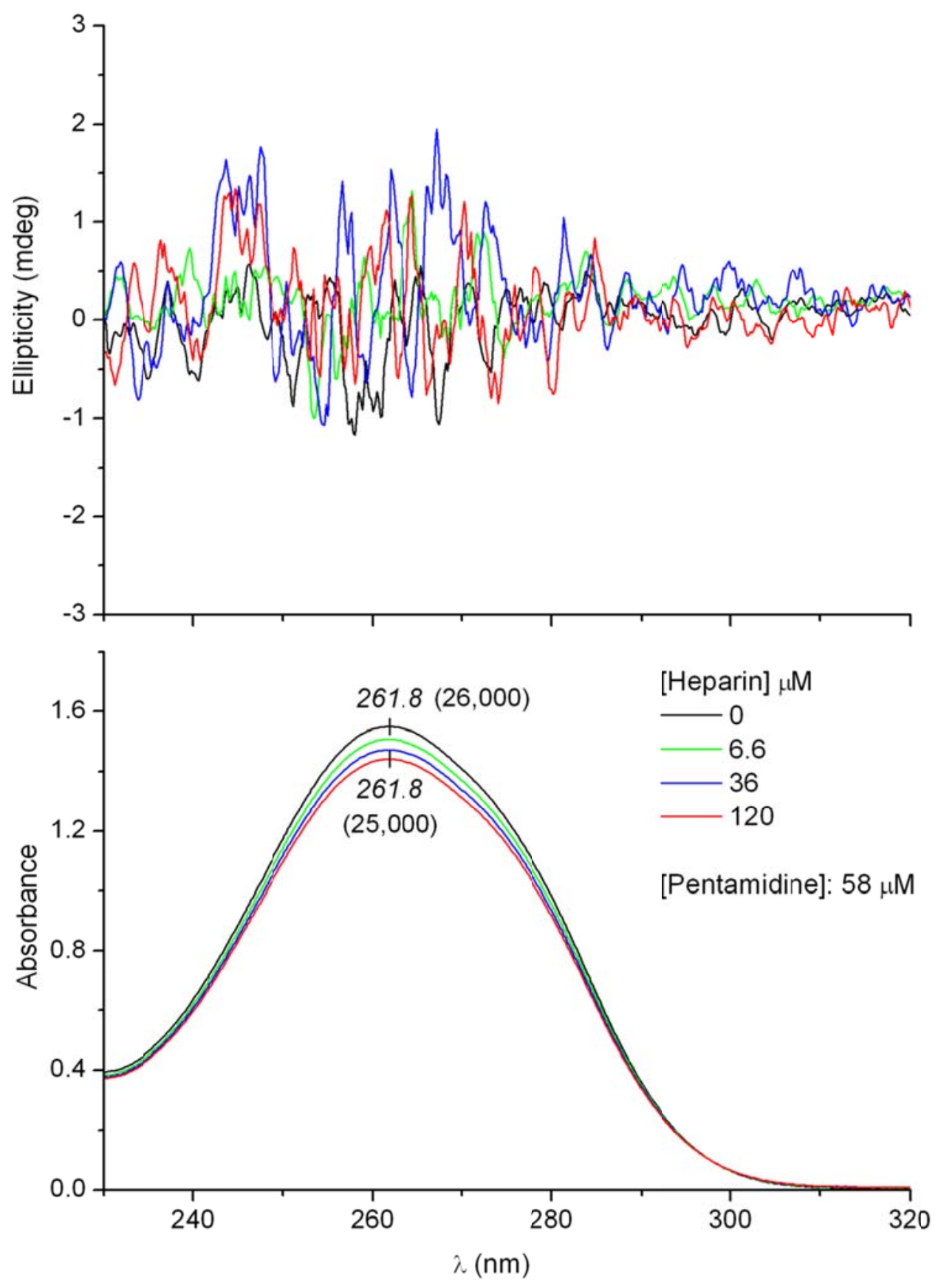

\section{Supplementary Figure 7}

$\mathrm{CD}$ and absorption spectra of $58 \mu \mathrm{M}$ pentamidine at increasing concentrations of heparin in pH 7.4 phosphate buffer with $140 \mathrm{mM} \mathrm{Na}^{+}\left(25{ }^{\circ} \mathrm{C}\right)$. Molar absorption cofficients $(\varepsilon)$ are shown in parentheses. 